\title{
Relativistic study of tautomerism and core electron binding energies of thio- and selenocytosine
}

\author{
Maria Barysz • Mariusz Klobukowski • \\ Jerzy Leszczynski
}

Received: 9 May 2011/Accepted: 5 October 2011/Published online: 4 June 2012

(C) The Author(s) 2012. This article is published with open access at Springerlink.com

\begin{abstract}
We applied the spin-free relativistic infiniteorder two-component method to calculate the core-electron binding energies of different tautomeric structures of thioand selenocytosine. The importance of relativistic effects in the ionization of $1 s$ electrons was studied for selenocytosine. The present method provides a reasonably simple and reliable computational tool for calculating the core electron binding energies of molecules containing heavy atoms. The method can also be used for characterization of different tautomeric structures of nucleic acid basis.
\end{abstract}

Keywords Core electron binding energies (CEBE) . Thiocytosine $\cdot$ Selenocytosine $\cdot$ Tautomers $\cdot$ Relativistic infinite-order two-component method (IOTC)

This paper is dedicated to Professor Małgorzata Witko in celebration of an anniversary in both private and scientific life.

\section{Barysz ( $\square)$}

Department of Quantum Chemistry, Institute of Chemistry,

Nicolaus Copernicus University, Gagarina 7, 87100 Toruń,

Poland

e-mail: teomjb@chem.umk.pl

M. Klobukowski

Department of Chemistry, University of Alberta,

Edmonton, AB T6G 2G2, Canada

e-mail: mariusz.klobukowski@ualberta.ca

J. Leszczynski

Department of Chemistry, Jackson State University,

Jackson, MS 39217, USA

e-mail: jerzy@icnanotox.org

\section{Introduction}

Tautomerism of the DNA bases is related to possibility of a transfer of hydrogen atoms between various heteroatoms (oxygens or nitrogens) of the bases and it has a vital effect on their properties and structures. It is also believed to be related to the DNA spontaneous mutations when rare tautomers are incorporated into genetic material. The core level photoemission and near X-ray photoabsorption spectra of different tautomers of nucleic acid basis in the gas phase can be measured and used for identification. The X-ray absorption structures near the ionization threshold are also closely related to the local electronic structure at the atom on which the localized excitation, i.e., the creation of the hole state, takes place. The spectra provide local probes of the charge distribution in molecules and of the ability of a molecule to accept charge. They can be related to and can provide insight into such chemical concepts and properties as proton affinities, reactivity, and regioselectivity of reactions [1-3].

An understanding of the physicochemical properties and tautomeric behaviour of the purine and pyrimidine bases of nucleic acids is of fundamental importance not only in relation to qualitative concepts of chemical bonding and physical chemistry but also in relation to molecular biology. The possible existence of one or more of the DNA bases in an unusual tautomeric form can increase the probability of mispairings of the purines with the pyrimidines and hence may lead to point mutation. Therefore several attempts, both experimental and theoretical, have been undertaken to study the physicochemical properties (including tautomerism phenomena) of DNA bases and their model systems. Several papers discussed the tautomerism of the nucleic acid bases (NABs) from experimental and theoretical points of view. The review of these studies goes beyond the scope of this article. 
Recent developments [4-6] in the high-resolution soft $\mathrm{X}$-ray photoelectron spectroscopy have enabled accurate and detailed experimental characterization of the coreelectron ionization processes. As the improved X-ray data become available, computational methods that can give reliable core electron binding energies (CEBE) of different molecular species become increasingly important.

The interplay between relativistic and electron correlation contributions to the electron binding energies depends on the ionized state. For holes of low energy, e.g., for singly ionized valence states, the proper treatment of the electron correlation contribution is of vital importance for the accuracy of the calculated CEBEs. In contrast, the deep core ionized states require that the relativistic effects be taken into account. Since most of the electron correlation contribution is (relatively) important only for valence (and perhaps next-to-valence) ionized states, the main problem in accurate calculations of CEBEs becomes the evaluation of the relativistic contribution to energy of the deep core states. This is not an easy task if one has in mind methods which can be used for large molecules involving heavy atoms.

Theoretical methods to calculate CEBE have a long history, dating from the early papers by Bagus and Schaefer [7, 8] to very accurate theoretical methods based on the density functional theory and developed by Chong [9-11] or the second and fourth-order algebraic-diagrammatic construction (ADC(2) and $\mathrm{ADC}(4))$ method for the polarization propagator [4-6, 12, 13]. Pseudopotential methods using effective integrals and Hamiltonian matrix elements have been quite successful in reproducing experimental spectra, see for example the recent $\triangle \mathrm{MP} 2 / \mathrm{MIX}$ method proposed by Shim et al. [14]. These methods can offer very accurate, fast, and effective way to calculate CEBEs, especially for bigger systems which cannot be handled by the all-electron ab initio approach. However, the availability of ab initio methods that are able to include both relativistic and many-electron effects is of great importance. In recent years four-component methods based on solving the Dirac-Hartree-Fock (DHF) configuration interaction $(\mathrm{CI})$ equations have been shown to be well suited for the study of core electron spectra [15].

During the past decade we have developed a new $\mathrm{ab}$ initio method which leads to enormous reduction of the computational effort and simultaneously recovers most of the relativistic effects which are accounted for within the Dirac formalism. This infinite-order two-component theory (IOTC) has been shown to completely recover the positive part of the Dirac spectrum for one-electron systems [1620]. For many-electron systems this method differs from DHF or correlated DHF schemes by the way of handling the two electron terms. However, except for very heavy systems, this difference appears to be negligible in comparison with other contributions (e.g., quantum electrodynamic (QED) corrections) which are usually neglected in DHF calculations of ionization potentials.

Of particular usefulness in large-scale applications of the IOTC approach in all methods for handling the electron correlation problem is the so-called one-component (scalar) IOTC approximation [16, 17, 19]. The structure of this method is essentially the same as that of any non-relativistic approach. Hence, the scalar IOTC theory can be easily used in the context of any correlated level approximation. The very simple algorithm which underlies this method makes it useful in investigations of systems for which the corresponding non-relativistic calculations can be carried out. There are two approximations involved: The first of them is the way of handling of two-electron terms. The second one is the use of the scalar approximation. In general IOTC (at any level of electron correlation treatment) is expected to work well.

The aim of the present paper is twofold. First, we shall demonstrate the reliability of the relativistic infinite order one-component approach (IOTC) in predicting CEBE of selected tautomeric structures of thio- and selenocytosine. Second, we shall discuss the possibility of using the relativistic quantum-chemical calculations at the applied level to identify the species for which experimental data are still elusive (e.g., selenocytosine).

\section{Computational methodology}

The main objective of our investigations is the study of characteristics of tautomeric forms of the thio- and selenocytosine. A knowledge of the relative stabilities of the tautomeric forms and the tautomeric identification is important from the point of view of experimental chemistry and medicine. The core level photoemission and near-edge $\mathrm{X}$-ray photoabsorption spectra together with computational methods can provide a good approach for the identification and characterization of different tautomeric structures of the nucleic acid bases. The subject is not new and significant developments in the experimental and the computational methods [4-6, 14, 21-23] took place in recent years. In the description of the inner shell ionization the relativistic effects are very important. Obviously, the experimental results contain relativistic effects. Most theoretical methods that have been used to calculate the CEBE in molecules are non-relativistic and the results are usually shifted by certain parametrical values to include the relativistic corrections, for example using the empiricallyderived formula due to Chong [10].

The quantum chemical calculations for systems containing heavy atoms require that the effects of relativity be included. The basic theory underlying all methods of 
relativistic quantum chemistry is essentially present in the Dirac equation [24]. The Dirac theory is formulated in terms of four-component functions (spinors) which makes it differ substantially from the non-relativistic approaches. Although the four-component molecular relativistic DiracCoulomb electronic structure calculations are possible, the cost of such calculations is still very high [25-27]. The reduction of this approach to the form resembling the usual methods of non-relativistic chemistry is known as the transition from four- to two-component approximation. The latter can be further spin-averaged leading to the socalled one-component relativistic methods whose formal structure is essentially the same as that of the methods of non-relativistic quantum chemistry; the principal difference amounts to the replacement of the usual energy operators by their approximate relativistic counterparts [17, 28-32]. The two-component formalism is actually not that much of an approximation as far as relativistic quantum chemistry is concerned. The ultimate goal of this formalism is to separate completely the positive and the negative energy solutions of the Dirac equation and to focus attention on the electronic solution only [17, 19, 28]. This can be accomplished in a rigorous and numerically accurate way, leading to what is termed the infinite two-component relativistic theory (IOTC) "for electrons only" [16, 19]. This is the approach that is used in the present study.

All calculations performed within the present study have been carried out with basis sets of uncontracted Gaussiantype orbitals (GTO). These basis sets are taken to be the uncontracted, truncated ANO-RCC basis [33] (atomic natural orbitals-relativistic correlation consistent sets of Gaussians) of the form [14s.9p.4d] for $\mathrm{C}$ and $\mathrm{N}$, [16s.12p.5d] for S, and [20s.17p.11d] for Se. The truncation of the original ANO-RCC basis (removal of the correlating f- and g-type Gaussian functions) was necessary due to the size of the studied tautomers.

The calculations of the CEBEs were carried out using the structural parameters of the tautomers in the gas phase that were optimized at the IOTC/MP2 level of theory. The ionization potential corresponding to the creation of a hole in the $1 s$ orbital of a closed shell $2 N$-electron system is obtained from:

$\operatorname{CEBE}(i)=E_{i}(2 N-1)-E(2 N)$,

where $E(2 N)$ and $E_{i}(2 N-1)$ are the energies of the $2 N$ and $(2 N-1)$-electron systems, respectively.

It has already been mentioned that the electron correlation contribution to CEBEs is vital for valence, and perhaps for the next-to-valence, ionized states. The deep core ionization is dominated by the difference in the relativistic contribution to the corresponding states. Thus, both of these contributions need to be taken into account if the method is to give realistic results whose quality does not depend on the type of the hole level. The major part of the relativistic effect on the energy levels is accounted for by the IOTC approach. The electron correlation contribution is calculated using the second-order Møller-Plesset perturbation theory (MP2) method of the electronic structure theory [34] which can be applied to any many-electron system.

The reference wave functions for neutral $2 N$-electron systems have been calculated in the IOTC self-consistent Hartree-Fock (HF) approximation. For the ionized species, the open-shell IOTC ROHF method has been employed. Once the SCF calculations were done, corrections for the many-electron correlation effects for both the neutral molecule and cation have been evaluated at the MP2 level of theory [34]. We have chosen the ROHF-MBPT variant $[35,36]$ of the open shell spin-restricted perturbation theory for the core hole calculations.

All the IOTC calculations reported in the present study were carried out using the GAMESS-US program [37]; the IOTC formalism has been added to GAMESS-US by one of us (M.B.). All computations done in the Department of Chemistry at the N. Copernicus University were performed on Intel-based Dell PowerEdge 2950 Server with dualprocessor Quad Core Intel Xeon X5460 and 32 GB of RAM. The $\triangle \mathrm{MP} 2 / \mathrm{MIX}$ calculations were carried out on Linux clusters at the Department of Chemistry and Department of Academic Information and Communication Technologies at the University of Alberta.

\section{Results and discussion}

\section{Pilot studies}

The infinite order two-component relativistic method has been already used for calculations of atomic core ionization potentials [38]. However, the agreement between the experimental data and theoretical IOTC results for the $1 s$ core ionization potentials is not always good enough and one may still see deviation of the calculated atomic IOTC results from the experimental values [38]. These deviations do not reflect the accuracy of the relativistic IOTC method but rather they may be attributed to the QED corrections: the Breit term, vacuum polarization, and self-energy terms which have not been taken into account in our calculations. These corrections were recently analyzed in the DiracHartree-Fock studies carried out by Słabkowska [39], who calculated the energy of transition involving the $1 s^{1} 2 p^{6}$ and $1 s^{2} 2 p^{5}$ states in the ion $\mathrm{Pd}^{+}(Z=46)$ and found that the contribution of the Breit term, vacuum polarization (VP), and self energy (SE) terms to the transition energy amounts to $-69.6 \mathrm{eV}$ (Breit $=-45.3 \mathrm{eV}, \mathrm{VP}=-27.9 \mathrm{eV}$, and $\mathrm{SE}=$ $+3.6 \mathrm{eV}$ ). The magnitude of the QED terms will strongly 
Fig. 1 Structure and numbering of atoms in cytosine, thiocytosine and selenocytosine tautomers $\mathrm{A}, \mathrm{C}$ and $\mathrm{D}(\mathrm{X}=\mathrm{O}$, $\mathrm{S}, \mathrm{Se})$

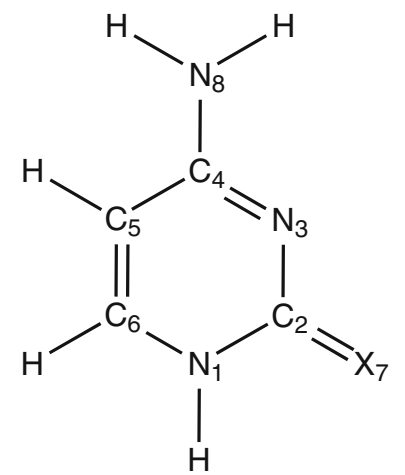

A<smiles>[Y]c1nc([Y]([H])[H])n[Z5]([H]):[Z]1[H]</smiles>

C

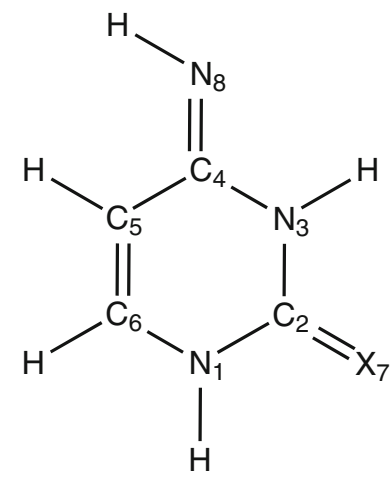

D depend on the atomic number of the studied element. For the carbon and nitrogen atoms these corrections should be very small. The interpolation of the data from Ref. [38] suggests the magnitude of QED corrections of about $2 \mathrm{eV}$ and $20 \mathrm{eV}$ for $\mathrm{S}$ and Se atoms, respectively. One should be aware of this fact when comparing results of the relativistic calculations with the experimental values. We do not know any molecular calculations of CEBE where these effects have been taken into account.

Availability of the IOTC formalism in GAMESS-US and the applications to the calculations of CEBE allowed us to further evaluate our previous method of calculating the CEBEs, the $\triangle \mathrm{MP} 2 / \mathrm{MIX}$ method [14]. That method was tested only for light atoms (carbon-fluorine). In order to carry out a meaningful comparison between the $\triangle \mathrm{MP} 2 /$ IOTC and $\triangle \mathrm{MP} 2 / \mathrm{MIX}$ methods, we slightly modified the original $\triangle \mathrm{MP} 2 / \mathrm{MIX}$ approach using the IOTC hamiltonian for the atom whose $1 s$ electron was ionized and for which an all-electron description was employed. The remaining atoms were represented by model core potentials which for the light atoms were parameterized using non-relativistic reference data [40].

In order to test the quality of the $\triangle \mathrm{MP} 2$-IOTC/ANO-RCC approach in molecular calculations we calculated the CEBEs of tautomer A of cytosine (Fig. 1) and compared the calculated values with the experimental data.

The relativistic effects in the studied tautomer are rather small, however at present it is the best available example. The final energies are shown in Table 1 . It is very pleasing to note that the CEBE values obtained within the present scalar $\triangle \mathrm{MP} 2-\mathrm{IOTC}$ method are close to the corresponding experimental data. The agreement between the old method $\triangle \mathrm{MP} 2 / \mathrm{MIX}[14]$ and the new results is satisfactory.

The CEBEs of cytosine have been studied recently experimentally and computationally by Feyer et al. [5]. In the study of Feyer et al., the calculated ADC(4) CEBEs were too high when compared to the experimental results [5] and they had to be shifted by $-1.55,-1.31$, and $-1.00 \mathrm{eV}$ for the $\mathrm{C}, \mathrm{N}$, and $\mathrm{O} 1 s$ ionization, respectively, to lower binding energies (see also the Table 1).
Table 1 Experimental and theoretical values of $1 s$ core electron binding energies in the tautomer $\mathrm{A}$ of cytosine

\begin{tabular}{lllll}
\hline & Exp. $^{\text {a }}$ & MP2-IOTC $^{\text {b }}$ & MP2/MIX $^{\text {b }}$ & ADC $(4)^{\text {c }}$ \\
\hline $\mathrm{O}_{7}$ & 536.5 & 537.03 & 536.818 & 536.22 \\
$\mathrm{~N}_{1}$ & 406.1 & 406.86 & 406.680 & 406.09 \\
$\mathrm{~N}_{3}$ & 404.5 & 404.72 & 404.479 & 404.04 \\
$\mathrm{~N}_{8}$ & 406.1 & 406.40 & 406.246 & 405.95 \\
$\mathrm{C}_{2}$ & 293.9 & 294.46 & 294.292 & 294.10 \\
$\mathrm{C}_{4}$ & 293.2 & 293.61 & 293.455 & 293.14 \\
$\mathrm{C}_{6}$ & 292.4 & 292.90 & 292.685 & 292.39 \\
$\mathrm{C}_{5}$ & 290.6 & 291.12 & 290.892 & 290.61 \\
\hline
\end{tabular}

All values in $\mathrm{eV}$

${ }^{a}$ Ref. [5]

b This work

c Shifted, Ref. [5]

Core electron binding energies of the tautomeric structures of thio- and selenocytosine

Tautomerism of thio- and selenocytosine has been studied less frequently than that of cytosine [41, 42]. However, thiocytosine and selenocytosine and their derivatives are of interest and importance because of their unusual biological properties. The thiocytosine tautomers base have been found in Escherichia coli tRNA [43-45]. Its nucleosides exhibit moderate inhibitory activity against vaccine and Epstein-Barr viruses in cell cultures and fluorinated 2-thiocytidine has shown potent antileukemic activity [4648]. Substitution of oxygen in a carbonyl group of cytosine by selenium causes changes in molecular properties associated with the $\mathrm{C}=\mathrm{X}$ group. Such changes play an important biological role [49, 50]. For instance, the ability of selenium to act as a redox catalyst has been an important factor in understanding the biological function of several selenoproteins, but still there is a lack of understanding of the role of selenium in biological structures. We anticipate that theoretical studies on such compounds may be helpful in understanding this role. Particularly, quantum-chemical 
calculations at the present level might be applied to identification of the species for which experimental data are still elusive (selenocytosine).

We studied three most stable tautomers, A, C, and D of thio- and selenocytosine (Fig. 1). The electronic ground state molecular structures of thio- and selenocytosine were obtained by means of full geometry optimization procedure at MP2-IOTC/ANO-RCC level of theory. At the optimized geometries, the total energies of the tautomers were -718 . $540059,-718.545984$, and -718.538398 au for thiocytosine and $-2748.655586,-2748.658809$, and -2748.653 316 au for selenocytosine tautomers, respectively. The substitution of oxygen in the carbonyl group of cytosine by sulfur or selenium does not change the ordering of stability of tautomers and tautomer $\mathrm{C}$ remains the most stable structure [14]. The calculated CEBE of the three tautomers are presented in Table 2 for thiocytosine and in Table 3 for selenocytosine and used to check the quality of the $\Delta \mathrm{MP} 2 / \mathrm{MIX}$ pseudopotential method [14].

Tables 2 and 3 reveal that the $1 s$ CEBE of sulfur and selenium atoms can be used for the identification of the tautomeric structures A, C, and D for both thio- and

Table 2 Theoretical values of $1 s$ core electron binding energies in tautomers of thiocytosine

\begin{tabular}{|c|c|c|c|c|c|c|}
\hline & \multicolumn{2}{|c|}{ Thiocytosine A } & \multicolumn{2}{|c|}{ Thiocytosine $\mathrm{C}$} & \multicolumn{2}{|c|}{ Thiocytosine D } \\
\hline & $\begin{array}{l}\text { MP2- } \\
\text { IOTC }\end{array}$ & $\begin{array}{l}\text { MP2/ } \\
\text { MIX }\end{array}$ & $\begin{array}{l}\text { MP2- } \\
\text { IOTC }\end{array}$ & $\begin{array}{l}\text { MP2/ } \\
\text { MIX }\end{array}$ & $\begin{array}{l}\text { MP2- } \\
\text { IOTC }\end{array}$ & $\begin{array}{l}\text { MP2/ } \\
\text { MIX }\end{array}$ \\
\hline $\mathrm{S}_{7}$ & 2476.52 & 2476.26 & 2479.16 & 2478.96 & 2477.44 & 2477.19 \\
\hline $\mathrm{N}_{1}$ & 406.98 & 406.76 & 404.99 & 404.77 & 407.34 & 407.12 \\
\hline $\mathrm{N}_{3}$ & 404.81 & 404.52 & 405.24 & 404.99 & 406.98 & 406.75 \\
\hline $\mathrm{N}_{8}$ & 406.54 & 406.34 & 406.40 & 406.23 & 404.36 & 404.12 \\
\hline $\mathrm{C}_{2}$ & 293.23 & 293.05 & 293.07 & 292.91 & 294.24 & 294.08 \\
\hline $\mathrm{C}_{4}$ & 293.61 & 293.43 & 293.39 & 293.18 & 293.82 & 293.59 \\
\hline $\mathrm{C}_{6}$ & 292.94 & 292.73 & 291.98 & 291.78 & 293.08 & 292.89 \\
\hline $\mathrm{C}_{5}$ & 291.16 & 290.90 & 290.93 & 290.70 & 291.28 & 291.06 \\
\hline
\end{tabular}

All values in $\mathrm{eV}$ selenocytosine. The tautomer $\mathrm{C}$ in both thio- and selenocytosine has always the largest value of $\mathrm{S}$ or Se $1 s \mathrm{CEBE}$, while the smallest value of the $1 s \mathrm{CEBE}$ is observed for the tautomer A. This observation can be confirmed by earlier theoretical and experimental results of the tautomeric structures of cytosine [5, 14]. Another interesting observation is that the values of the $1 s$ CEBEs of the light atoms in the seleno- and thiocytosine tautomers are almost the same for a given atom, except for the sulphur, selenium and $C_{2}$ atoms. For example, the values are: for $N_{1} 1 s 406.98$ and $406.99 \mathrm{eV}$, for $N_{3} 1 s 404.81$ and $404.81 \mathrm{eV}$, for $C_{4} 1 s 293.61$ and $293.59 \mathrm{eV}$, for thio- and selenocytosine A, respectively. Again, it is pleasing to observe a satisfactory agreement of the results obtained using the $\triangle \mathrm{MP} 2 / \mathrm{MIX}$ method with the present values.

The present results clearly support the idea of using calculated or experimental values of CEBEs for the characterization of the chemical or biological structures.

Role of relativistic effects

In Table 4 we compiled all data which show the importance of the relativistic and electron correlation effects on the calculated CEBE. These include the $1 s \mathrm{CEBE}$ results of the open-shell, Hartree-Fock (ROHF) and second order Møller-Plesset (MP2-RMP) calculations. In both cases, the non-relativistic and relativistic IOTC results are given and allow us to directly reveal the magnitude of relativistic contributions for different atoms. The HF-based method offers a simple way to estimate energies of different $\mathrm{CE}$ BEs. It does not take into account an electron correlation contribution to CEBEs. However, it gives the right order of magnitude of relativistic effects and on the basis of the HFtype calculations for the parent neutral system it indicates if these effects need to be included in higher-level calculations. According to the data of Table 4 the relativistic contributions become increasingly important for the heaviest atom, selenium, for which the non-relativistic NR MP2 CEBE result for the $1 s$ level would be too small by
Table 3 Theoretical values of $1 s$ core electron binding energies in tautomers of selenocytosine

All values in $\mathrm{eV}$

\begin{tabular}{|c|c|c|c|c|c|c|}
\hline & \multicolumn{2}{|c|}{ Selenocytosine A } & \multicolumn{2}{|c|}{ Selenocytosine C } & \multicolumn{2}{|c|}{ Selenocytosine D } \\
\hline & MP2-IOTC & MP2/MIX & MP2-IOTC & MP2/MIX & MP2-IOTC & MP2/MIX \\
\hline $\mathrm{Se}_{7}$ & 12682.19 & 12682.00 & 12684.64 & 12684.50 & 12683.07 & 12682.90 \\
\hline $\mathrm{N}_{1}$ & 406.99 & 406.77 & 404.96 & 404.74 & 407.34 & 407.13 \\
\hline $\mathrm{N}_{3}$ & 404.81 & 404.53 & 405.20 & 404.98 & 406.96 & 406.75 \\
\hline $\mathrm{N}_{8}$ & 406.55 & 406.36 & 406.38 & 406.23 & 404.35 & 404.13 \\
\hline $\mathrm{C}_{2}$ & 292.92 & 292.74 & 293.24 & 292.61 & 293.89 & 293.74 \\
\hline $\mathrm{C}_{4}$ & 293.59 & 293.38 & 293.32 & 293.16 & 293.77 & 293.59 \\
\hline $\mathrm{C}_{6}$ & 292.95 & 292.74 & 291.91 & 291.75 & 293.09 & 292.89 \\
\hline $\mathrm{C}_{5}$ & 291.16 & 290.89 & 290.81 & 290.70 & 291.25 & 291.05 \\
\hline
\end{tabular}


Table 4 Theoretical values of non-relativistic and relativistic $1 s$ CEBE in tautomer A of selenocytosine

\begin{tabular}{|c|c|c|c|c|c|c|c|c|}
\hline & NR HF & IOTC HF & NR MP2 & IOTC MP2 & $\Delta_{\text {rel }}^{\mathrm{HF}}$ & $\Delta_{\mathrm{rel}}^{\mathrm{MP2}}$ & $\Delta_{\text {corr }}^{\mathrm{NREL}}$ & $\Delta_{\text {corr }}^{\mathrm{IOTC}}$ \\
\hline $\mathrm{Se}_{7}$ & 12484.30 & 12679.45 & 12495.31 & 12682.19 & 195.15 & 186.88 & 11.01 & 2.74 \\
\hline $\mathrm{N}_{1}$ & 406.36 & 406.54 & 406.81 & 406.99 & 0.18 & 0.18 & 0.45 & 0.45 \\
\hline $\mathrm{N}_{3}$ & 403.90 & 404.08 & 404.63 & 404.81 & 0.18 & 0.18 & 0.73 & 0.73 \\
\hline $\mathrm{N}_{8}$ & 405.99 & 406.18 & 406.36 & 406.55 & 0.19 & 0.19 & 0.37 & 0.37 \\
\hline $\mathrm{C}_{2}$ & 293.62 & 293.68 & 292.85 & 292.92 & 0.06 & 0.07 & -0.77 & -0.76 \\
\hline $\mathrm{C}_{4}$ & 294.22 & 294.29 & 293.52 & 293.59 & 0.07 & 0.07 & -0.70 & -0.70 \\
\hline $\mathrm{C}_{6}$ & 293.22 & 293.30 & 292.86 & 292.95 & 0.08 & 0.09 & -0.36 & -0.35 \\
\hline $\mathrm{C}_{5}$ & 290.79 & 290.87 & 291.05 & 291.16 & 0.08 & 0.11 & 0.26 & 0.29 \\
\hline
\end{tabular}

All values in $\mathrm{eV}$;

$\Delta_{r e l}^{H F}=\mathrm{CEBE}(\mathrm{IOTC} \mathrm{HF})-\mathrm{CEBE}(\mathrm{NR} \mathrm{HF})$;

$\Delta_{r e l}^{M P 2}=\mathrm{CEBE}(\mathrm{IOTC}$ MP2) $-\mathrm{CEBE}(\mathrm{NR}$ MP2);

$\Delta_{\text {corr }}^{N R E L}=\mathrm{CEBE}(\mathrm{NR} \mathrm{MP} 2)-\mathrm{CEBE}(\mathrm{NR} \mathrm{HF})$;

$\Delta_{\text {corr }}^{\text {IOTC }}=\mathrm{CEBE}(\mathrm{IOTC} \mathrm{MP} 2)-\mathrm{CEBE}(\mathrm{IOTC} \mathrm{HF})$

$186.88 \mathrm{eV}$ relative to the IOTC MP2 value. The corresponding NR HF and IOTC HF CEBE differ by $195.15 \mathrm{eV}$.

For carbon and nitrogen atoms the relativistic contribution to the CEBE are small (about $0.1-0.2 \mathrm{eV}$ ) but not negligible if one aims at chemical accuracy. As can be seen in Table 4, these corrections do not change for a given light atom when either the position of the atom is changed $\left(\mathrm{C}_{2}\right.$ vs. $\mathrm{C}_{4}$ ) or the level of theory (HF vs. MP2). Consequently, we may use a constant relativistic correction for the light atoms in non-relativistic calculations. While the empirical formula of Chong depends on the value of CEBE [10], in recent calibration work by Besley et al. [51] a constant relativistic correction of $0.1,0.21,0.36$, and $0.63 \mathrm{eV}$ is added for the $1 s$ orbitals of $\mathrm{C}, \mathrm{N}, \mathrm{O}$, and $\mathrm{F}$, respectively. We computed the relativistic corrections to the $1 s$ CEBEs for the $\mathrm{C}, \mathrm{N}, \mathrm{O}$, and $\mathrm{F}$ at the Hartree-Fock level in the molecules of methane, ammonia, water, and hydrogen fluride. Using the same all-electron basis set that was used in the present study we obtained the values of $0.101,0.184$, 0.334 , and $0.558 \mathrm{eV}$ for $\mathrm{C}, \mathrm{N}, \mathrm{O}$, and $\mathrm{F}$, respectively, fairly close to the values used by Besley et al.

The MP2 CEBE data which include the electron correlation effects show that the latter are not as important as the relativistic contributions. The experimental data for 1s CEBE of selenocytosine are not known.

\section{Conclusion}

We presented the first application of the IOTC method to calculate the $1 s$ CEBE in molecules. The relativistic calculations based on the spin-free part of the IOTC Hamiltonian were used to calculate the $1 s$ core ionization potentials of tautomeric structure of selenocytosine and thiocytosine. The comparison of the pseudopotential
$\Delta \mathrm{MP} 2 / \mathrm{MIX}$ results with the $\Delta \mathrm{MP} 2 / \mathrm{IOTC}$ data shows that the $\triangle \mathrm{MP} 2 / \mathrm{MIX}$ method can be reliably used for calculations of ionization potentials in molecules. It is interesting to notice (see the Tables 1,2 and 3) that the deviations of the MP2-IOTC from $\triangle \mathrm{MP} 2 / \mathrm{MIX}$ results are almost constant in all cases, and are about $0.2 \mathrm{eV}$. We may then expect similar results for other nucleic acid basis as well. In addition, we have shown that CEBEs can be used for characterization of different tautomeric structures of nucleic acid bases.

Acknowledgments MK thank the Natural Sciences and Engineering Research Council of Canada for the support of the present project under Research Grant No. G121210414. At JSU this study was supported by the NSF CREST. This study was supported by the NSF Nanotoxicity CREST Center Grant No. HRD-0833178.

Open Access This article is distributed under the terms of the Creative Commons Attribution License which permits any use, distribution, and reproduction in any medium, provided the original author(s) and the source are credited.

\section{References}

1. Siegbahn K, Nordling C, Johansson G (1969) ESCA applied to free molecules. North-Holland, Amsterdam

2. Siegbahn K, Fahlman C, Nordberg R, Hamrin K, Hedman J, Johansson G, Bergmark T, Karlsson SE, Lindgren L, Lindberg B (1967) ESCA atomic, molecular and solid state structure studied by means of electron spectroscopy, Uppsala

3. Bakke AA, Chen AW, Jolly WL (1980) J Electron Spectrosc Relat Phenom 20:33

4. Plekan O, Feyer V, Richter R, Coreno M, deSimone M, Prince KC, Trofimov AB, Gromov EV, Zaytseva IL, Schirmer J (2008) Chem Phys 347:360

5. Feyer V, Plekan O, Richter R, Coreno M, Vall-Llosera G, Prince KC, Trofimov AB, Zaytseva IL, Moskovskaya TE, Gromov EV, Schirmer J (2009) Chem Phys 113:5736 
6. Plekan O, Feyer V, Richter R, Coreno M, Vall-Llosera G, Prince KC, Trofimov AB, Zaytseva IL, Moskovskaya TE, Gromov EV, Schirmer J (2009) J Chem Phys 113:9376

7. Bagus PS, Schaefer HF (1971) J Chem Phys 55:1474

8. Bagus PS, Schaefer HF (1972) J. Chem. Phys. 56:224

9. Chong DP (1995) Chem Phys Lett 232:486

10. Chong DP (1995) J Chem Phys 103:1842

11. Chong DP (1997) Recent advances in density functional methods. Part II. World Scientific, Singapore

12. Schirmer J (1982) Phys Rev A 26:2395

13. Trofimov AB, Schirmer J (1995) J Phys B 28:2299

14. Shim J, Klobukowski M, Barysz M, Leszczyński J (2011) Phys Chem Chem Phys 13:5703

15. Broer R (2010) In: Barysz M, Ishikawa Y (eds) Challenges and advances in computational chemistry and physics, vol 10. Relativistic methods for chemists. Springer, London

16. Barysz M, Sadlej AJ (2002) J Chem Phys 116:2696

17. Barysz M (2003) In: Kaldor U, Wilson S (eds) Progress in theoretical chemistry and physics. Theoretical chemistry and physics of heavy and superheavy elements. Kluwer, London

18. Barysz M, Mentel L, Leszczyński J (2009) J Chem Phys 130: 164114

19. Barysz M (2010) In: Barysz M, Ishikawa Y (eds) Challenges and advances in computational chemistry and physics, vol 10. Relativistic methods for chemists. Springer, London

20. Kȩdziera D, Barysz M (2004) Chem Phys Lett 393:521

21. ADF Program System Technical report, Scientific Computing and Modelling NV, Vrije Universiteit, Theoretical Chemistry, De Boelelaan 1083, 1081 HV Amsterdam, The Netherlands (2000)

22. Chong DP (2007) J Electron Spectrosc Relat Phenom 159:94

23. Chong DP (2000) J Electron Spectrosc Relat Phenom 106:1

24. Moss RE (1973) Advanced molecular quantum mechanics. Chapman and Hall, London

25. Quiney HM, Grant IP, Wilson S (1989) Lecture notes in chemistry 52:331

26. Wilson S (2001) J Mol Struct (Theochem) 547:279

27. Saue T, Visscher L (2003) In: Kaldor U, Wilson S (eds) Theoretical chemistry and physics of heavy and superheavy elements, p. 211. Kluwer, Dordrecht

28. Barysz M, Sadlej AJ (2001) J Mol Struct (Theochem) 573:181
29. Hess BA (1985) Phys Rev A 32:756

30. Hess BA (1986) Phys Rev A 33:3742

31. Wolf A, Reiher M, Hess M (2002) In: Schwerdtfeger P (eds) The relativistic electronic structure theory. Part 1. Fundamentals, p. 622. Elsevier, Amsterdam

32. Reiher M, Wolf A (2009) Relativistic quantum chemistry: the fundamental theory of molecular science. Wiley, Weinheim

33. Roos BO, Lindh R, Malmqvist PO, Veryazov V, Widmark PO (2005) J Phys Chem A 108:2851

34. Møller Ch, Plesset MS (1934) Phys Rev 46:207

35. Knowles PJ, Andrews JS, Amos RD, Handy NC, Pople JA (1991) Chem Phys Lett 186:130

36. Lauderdale WJ, Stanton JF, Gauss J, Watts JD, Bartlett RJ (1991) Chem Phys Lett 187:213

37. Schmidt MW, Baldridge KK, Boatz JA, Elbert ST, Gordon MS, Jensen JH, Koseki S, Matsunaga N, Nguyen KA, Su S, Windus TL, Dupuis M, Montgomery JA (1993) J Comput Chem 14:1347

38. Barysz M, Leszczyński J (2007) J Chem Phys 126:154106

39. Słabkowska K (2011) private information, N. Copernicus University

40. Miyoshi E, Mori H, Hirayama R, Osanai Y, Noro T, Honda H, Klobukowski M (2005) J Chem Phys 122:074104

41. Kwiatkowski JS, Leszczyński J (1996) J Phys Chem 100:941

42. Venkateswarlu D, Leszczyński J (1998) J Phys Chem 102:6161

43. Carbon J, David H, Studier MH (1968) Science 161:1146

44. Gauss DH, Spinnzi MS (1983) Nucleic Acid Res 11:1

45. Yamada Y, Saneyoshi S, Nishimura S, Ishikura H (1970) FEBS Lett 7:207

46. Ruyle WV, Shen TY (1967) J Med Chem 10:331

47. Beauchamp LM, Serling BL, Kesley JE, Biron KK, Collins P, Selway J, Lin LC, Schaeffer HJ (1988) J Med Chem 31:144

48. Balińska M, Bretner M, Kulikowski T, Rode W, Shugar D (1992) In: 7th NCI-EORTC symposium on new drugs in cancer therapy, Amsterdam, p. 125

49. Zingaro RA (1974) In: Cooper WC (eds) Selenium. Van Nostrand Reinhold, New York

50. Patai S (1986) In: Rappoport Z (eds) The chemistry of organic selenium and tellurium compounds, vol 1. Wiley, New York

51. Besley NA, Gilbert ATB, Gill PMW (2009) J Chem Phys 130: 124308 